\title{
Erratum: Physically feasible three-level transitionless quantum driving with multiple Schrödinger
} dynamics [Phys. Rev. A 93, 052324 (2016)]

\author{
Xue-Ke Song (宋学科), Qing Ai (艾清), Jing Qiu (邱静), and Fu-Guo Deng (邓富国) \\ (Received 8 July 2016; published 21 July 2016)
}

DOI: 10.1103/PhysRevA.94.019905

Equation (17) in Sec. II B should be

$$
H_{M}(t)=\left(\begin{array}{ccc}
0 & 0 & \eta \cos \theta+V_{1} \\
0 & 0 & \eta \sin \theta-V_{2} \\
\eta \cos \theta+V_{1} & \eta \sin \theta-V_{2} & 0
\end{array}\right),
$$

where $V_{1}=4 \sin \theta(\dot{\eta} \dot{\theta}-\eta \ddot{\theta}) / R^{2}, V_{2}=4 \cos \theta(\dot{\eta} \dot{\theta}-\eta \ddot{\theta}) / R^{2}, \dot{\eta}=\left(\eta_{1} \dot{\eta}_{1}+\eta_{2} \dot{\eta_{2}}\right) / \eta$, and $\ddot{\theta}=\left[\left(\ddot{\eta}_{1} \eta_{2}-\eta_{1} \ddot{\eta}_{2}\right) \eta-2 \dot{\eta}\left(\dot{\eta}_{1} \eta_{2}-\eta_{1} \dot{\eta}_{2}\right)\right] / \eta^{3}$. This correction does not affect any other part of the paper, leaving our discussion unchanged. 